\title{
The Basis Number of Quadruple Join of Graphs
}

\section{Ghassan T. Marougi}

College of Computer Sciences and Mathematics

University of Mosul

Received on: 23/02/2011

Accepted on: 04/04/2011

\section{ABSTRACT}

The basis number, $b(G)$, of a graph $G$ is defined to be the smallest positive integer $\mathrm{k}$ such that $\mathrm{G}$ has a $\mathrm{k}$-fold basis for its cycle space. We investigate an upper bound for $b\left(G_{1}+G_{2}+G_{3}+G_{4}\right)$.It is proved that, if $G_{1}, G_{2}, G_{3}$ and $G_{4}$ are connected vertex-disjoint graphs and each has a spanning tree of vertex degree not more than 4 , then $b\left(G_{1}+G_{2}+G_{3}+G_{4}\right) \leq \max \left\{4, \mathrm{~b}\left(\mathrm{G}_{1}\right)+1, \mathrm{~b}\left(\mathrm{G}_{2}\right)+2, \mathrm{~b}\left(\mathrm{G}_{3}\right)+2, \mathrm{~b}\left(\mathrm{G}_{4}\right)+1\right\}$.

The basis number of quadruple join of paths, are studied. It is proved that $b\left(P_{\mathrm{m}}+P_{n}+P_{p}+P_{t}\right)=4, \quad \forall m, \mathrm{t} \geq 5$ and $n, p \geq 6$.

Keywords: Basis number, Cycle space.

$$
\begin{aligned}
& \text { العدد الأساس للاتصال الرباعي للبيانات } \\
& \text { رغد عبد العزيز مصطفى } \\
& \text { غسان طوبيا مروكي } \\
& \text { كلية علوم الحاسوب والرياضيات } \\
& \text { جامعة الموصل الموب ولكال } \\
& \text { تاريخ قبول البحث: 2011/04/04 } \\
& \text { تاريخ استلام البحث: 2011/02/23 }
\end{aligned}
$$

\section{الملخص}


في السنوات الأخيرة زاد الاهتمام بالعدد الأساس للبيانات, نشير للقارئ الرجوع إلى البحوث[4], [5], [6],

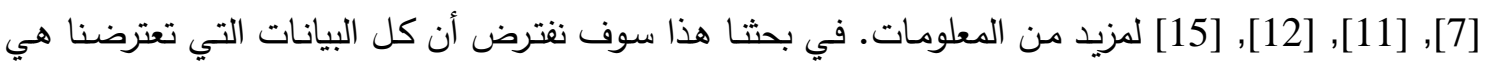
بيانات منتهية وغير موجهة وبسيطة؛ بالنسبة للمصطلحات غير المعرفة (راجع المصدرين [9] و [10]).

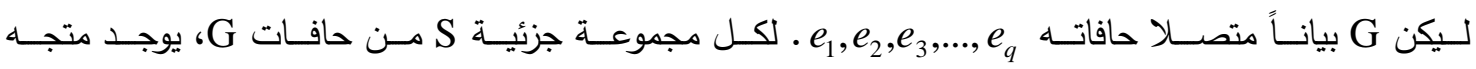

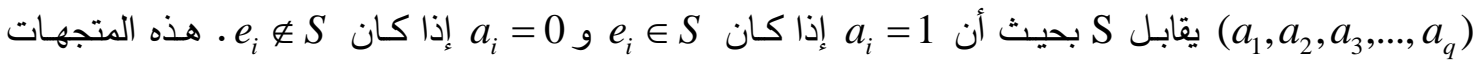

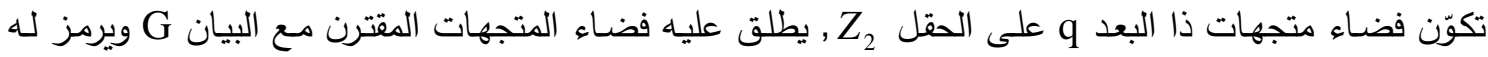

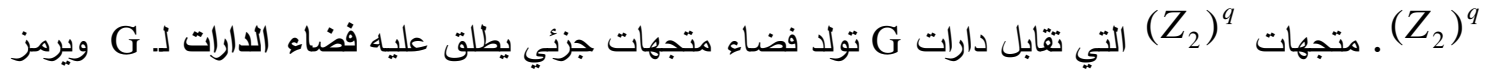

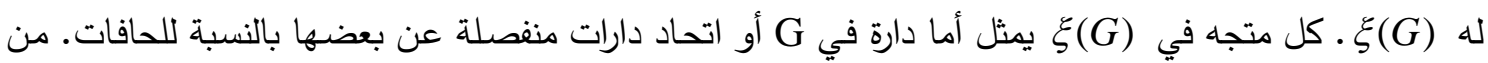
النتائج المعروفة في نظريـة البيان أن بعد فضاء الدارات لبيان متصل G) G(G) هو عدد رؤوس G و q عدد حافاته.

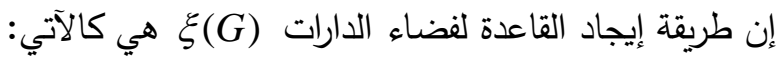
لتكن T شجرة مولدة في G؛ إذا كانت e حافة تتنمي إلى G-T عندئذ, T+e يحتوي على دارة واحدة فقط ولتكن

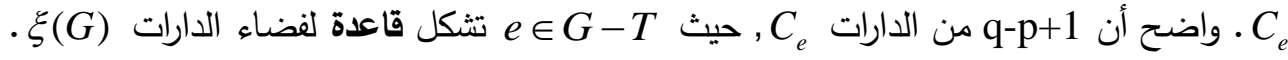

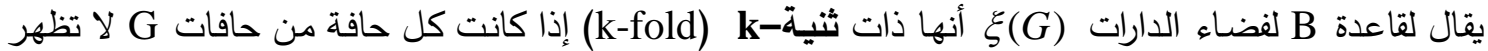
في أكثر من k من المرات في الدارات التي تقابل المتجهات في القاعدة B. ويعرف العدد الأساس (basis number) لبيان G بأنه العدد الصحيح الأصغر k بحيث أن (G) ك له له قاعدة ذات

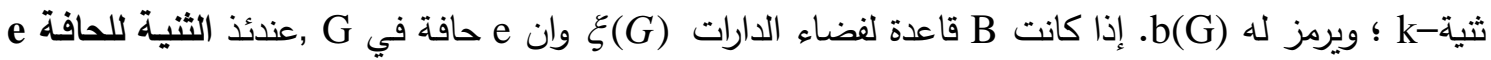
في B يرمز لها (e)

تعريف (1):

ليكن

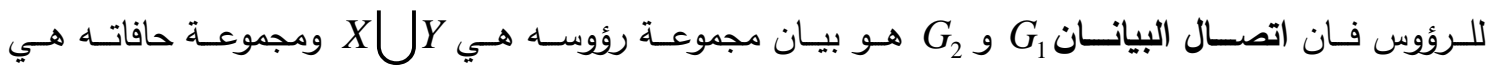
ويرمز له $E_{1} \bigcup E_{2} \bigcup\{x y \mid x \in X, y \in Y\}$ $X \bigcup Y \bigcup Z$

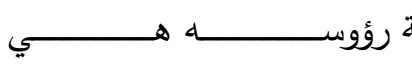

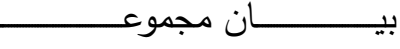
هـ نعرف الاتصـال الربـاعي حافاتسـه هـ فثثلاً

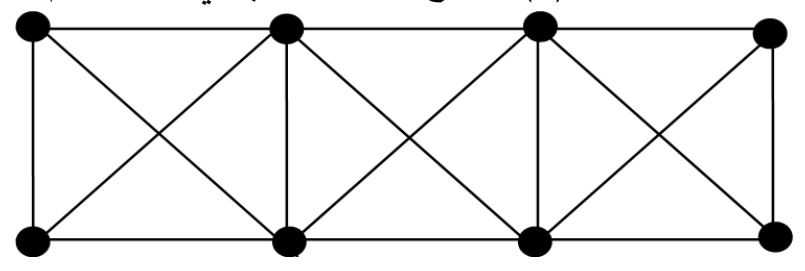

$$
K_{2}+K_{2}+K_{2}+K_{2} \cdot(\mathbf{1}) \text { شكل }
$$


وبطريقة مشابهة نعرف اتصال n من البيانات،

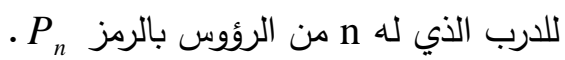
في عام 1937, برهن مكلين [13] أول مبرهنة مهمة في هذا الموضوع وهي تتص على "يكون البيان G مستويا إذا

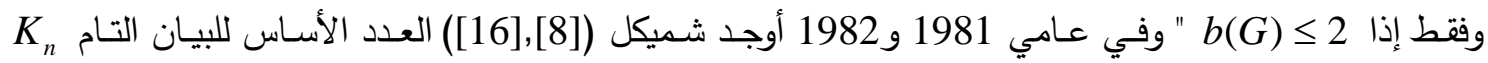
والبيان الثنائي التجزئة التام $K_{m, n}$ ومكعبn.n. العدد الأساس للجداء ألمعاجمي للبيانات تم حسابه في [1], كذلك العدد الأساس للجداء الديكارتي لبعض البيانات تم دراسته في [2]، ودرس علي [3] العدد الأساس لاتصال بيانين

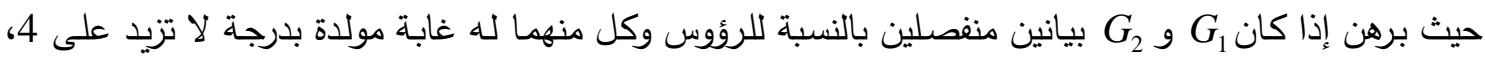
$b\left(G_{1}+G_{2}\right) \leq \max \left\{4, \mathrm{~b}\left(\mathrm{G}_{1}\right)+1, \mathrm{~b}\left(\mathrm{G}_{2}\right)+1\right\}$.

وفي [15] تم دراسة العدد الأساس للاتصال الثلاثي للبيانات. وفي بحثنا هذا سوف ندرس العدد الأساس للاتصال الرباعي للبيانات، حيث برهنا $b\left(G_{1}+G_{2}+G_{3}+G_{4}\right) \leq \max \left\{4, \mathrm{~b}\left(\mathrm{G}_{1}\right)+1, \mathrm{~b}\left(\mathrm{G}_{2}\right)+2, \mathrm{~b}\left(\mathrm{G}_{3}\right)+2, \mathrm{~b}\left(\mathrm{G}_{4}\right)+1\right\}$

$b\left(P_{\mathrm{m}}+P_{n}+P_{p}+P_{t}\right)=4, \quad \forall m, \mathrm{t} \geq 5$ and $n, p \geq 6$.

كما برهنا

$$
\text { : } G_{1}+G_{2}+G_{3}+G_{4} \text { ـ العدد الأساس }
$$

$U=\left\{u_{1}, u_{2}, \ldots, u_{t}\right\}, Z=\left\{z_{1}, z_{2}, \ldots, z_{p}\right\}, Y=\left\{y_{1}, y_{2}, \ldots, y_{n}\right\}, X=\left\{x_{1}, x_{2}, \ldots, x_{m}\right\}$ نفـــــ ونفرض أن Y,X

$B_{r}\left(K_{m, n}\right)=\left\{x_{i} y_{j} x_{i+1} y_{j+1} \mid i=1,2, \ldots, m-1\right.$ and $\left.j=1,2, \ldots, n-1\right\}$,

$B_{r}\left(K_{n, p}\right)=\left\{y_{i} z_{j} y_{i+1} z_{j+1} \mid i=1,2, \ldots, n-1\right.$ and $\left.j=1,2, \ldots, p-1\right\}$,

$B_{r}\left(K_{p, t}\right)=\left\{z_{i} u_{j} z_{i+1} u_{j+1} \mid i=1,2, \ldots, p-1\right.$ and $\left.j=1,2, \ldots, t-1\right\}$.

هي القواعد المطلوبة (required bases) لـ ( ) أن 4 أن $A_{1}=\left\{x_{1} y_{1}, x_{1} y_{n}, x_{m} y_{1}, x_{m} y_{n}\right\} \bigcup\left\{y_{1} z_{1}, y_{1} z_{p}, y_{n} z_{1}, y_{n} z_{p}\right\} \bigcup\left\{z_{1} u_{1}, z_{1} u_{t}, z_{p} u_{1}, z_{p} u_{t}\right\}$,

هـي مجموعـة حافـات و

$A_{2}=\left(\left\{x_{1} y_{j}, x_{m} y_{j} \mid j=2,3, \ldots, n-1\right\} \bigcup\left\{x_{i} y_{1}, x_{i} y_{n} \mid i=2,3, \ldots, m-1\right\}\right)$

$\bigcup\left(\left\{y_{1} z_{j}, y_{n} z_{j} \mid j=2,3, \ldots, p-1\right\} \bigcup\left\{y_{j} z_{1}, y_{j} z_{p} \mid j=2,3, \ldots, n-1\right\}\right)$

$\bigcup\left(\left\{z_{1} u_{j}, z_{p} u_{j} \mid j=2,3, \ldots, t-1\right\} \bigcup\left\{z_{j} u_{1}, z_{j} u_{t} \mid j=2,3, \ldots, p-1\right\}\right)$.

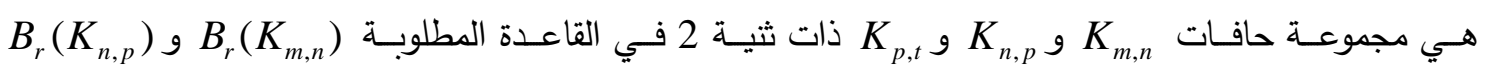

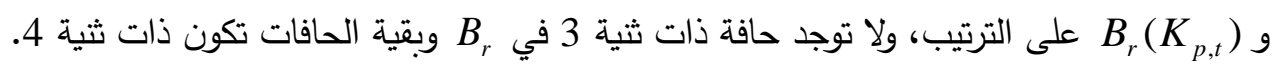
الآن سوف ندرس العدد الأساس للاتصال الرباعي للبيانات، أي $)$

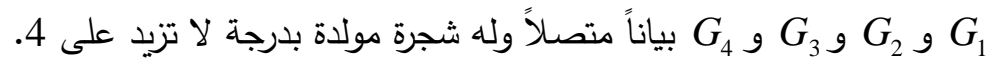


لـتكن لـ

$\operatorname{deg}_{T_{i}} u \leq 4, \forall u \in V\left(T_{i}\right)$

بما أن كل شجرة تحتوي على رأسين في الأقل بدرجة واحد فإننا نفرض أن $\operatorname{deg}_{T_{1}}\left(x_{1}\right)=\operatorname{deg}_{T_{1}}\left(x_{m}\right)=\operatorname{deg}_{T_{2}}\left(y_{1}\right)=\operatorname{deg}_{T_{2}}\left(y_{n}\right)=\operatorname{deg}_{T_{3}}\left(z_{1}\right)=\operatorname{deg}_{T_{3}}\left(z_{p}\right)=$ $\operatorname{deg}_{T_{4}}\left(u_{1}\right)=\operatorname{deg}_{T_{4}}\left(u_{t}\right)=1$

الآن لكل حافة $x_{i} x_{j} y_{n} x_{i} x_{i} x_{j} y_{1}$ في الثجرة $T_{1}$ سوف نختار دارة ثلاثية واحدة وواحدة فقط بالصيغة وهذا يكون بالطريقة التالية:

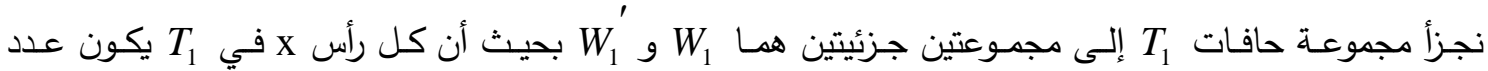
الحافات الواقعة عليه والتي تتنمي إلى نفس المجموعة الجزئية لا تزيد على 2.

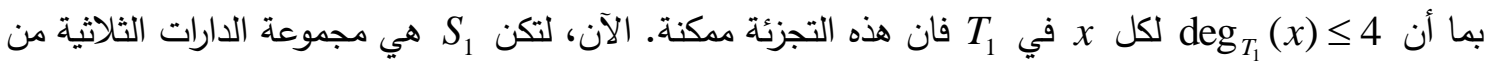
T المعرفة بالثكل الآتي: $S_{1}=\left\{x_{i} x_{j} y \mid x_{i} x_{j} \in E\left(T_{1}\right), y=y_{1}\right.$ if $x_{i} x_{j} \in W_{1}$ and $y=y_{n}$ if $x_{i} x_{j} \in W_{1}^{\prime}$

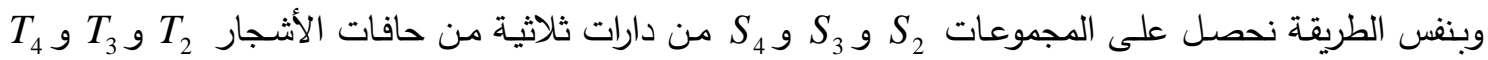
من البيانات $S_{2}=\left\{x y_{i} y_{j}, y_{i} y_{j} z \mid y_{i} y_{j} \in E\left(T_{2}\right), x=x_{1}, z=z_{1}\right.$ if $y_{i} y_{j} \in W_{2}$ and $x=x_{m}, z=z_{p}$ if $\left.y_{i} y_{j} \in W_{2}^{\prime}\right\}$, $S_{3}=\left\{y z_{i} z_{j}, z_{i} z_{j} u \mid z_{i} z_{j} \in E\left(T_{3}\right), y=y_{1}, u=u_{1}\right.$ if $z_{i} z_{j} \in W_{3}$ and $y=y_{n}, u=u_{t}$ if $\left.z_{i} z_{j} \in W_{3}^{\prime}\right\}$ and $S_{4}=\left\{z u_{i} u_{j} \mid u_{i} u_{j} \in E\left(T_{4}\right), z=z_{1}\right.$ if $u_{i} u_{j} \in W_{4}$ and $z=z_{p}$ if $\left.u_{i} u_{j} \in W_{4}^{\prime}\right\}$.

واضح أن 1)

كل حافة من الحافات وكل حافة من الحافات وكل حافة من الحافات وكل حافة من الحافات وكل حافة من الحافات وأخيرا كل حافة من الحافات وألان، نحن مستعدون لإثبات المبرهنة الأساسية في هذا البحث.

مبرهنة (1):

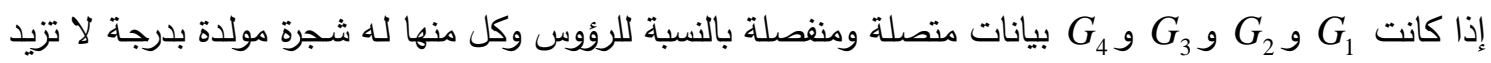
عن 4، فان $b\left(G_{1}+G_{2}+G_{3}+G_{4}\right) \leq \max \left\{4, \mathrm{~b}\left(\mathrm{G}_{1}\right)+1, \mathrm{~b}\left(\mathrm{G}_{2}\right)+2, \mathrm{~b}\left(\mathrm{G}_{3}\right)+2, \mathrm{~b}\left(\mathrm{G}_{4}\right)+1\right\}$. 
لتكن $G_{1}$ ل للبيانـات

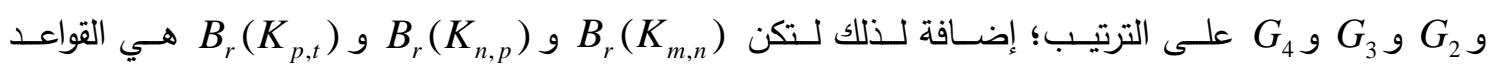
المطلوبة للبيانات

$$
\begin{aligned}
& B=B_{r}\left(G_{1}\right) \bigcup B_{r}\left(G_{2}\right) \bigcup B_{r}\left(G_{3}\right) \bigcup B_{r}\left(G_{4}\right) \bigcup B_{r}\left(K_{m, n}\right) \bigcup B_{r}\left(K_{n, p}\right) \bigcup B_{r}\left(K_{p, t}\right) \\
& \bigcup S_{1} \bigcup S_{2} \bigcup S_{3} \bigcup S_{4} \\
& \text { والآن سوف نبرهن أن B هي قاعدة لفضاء الدارات } \\
& |B|=\operatorname{dim} \xi\left(G_{1}\right)+\operatorname{dim} \xi\left(G_{2}\right)+\operatorname{dim} \xi\left(G_{3}\right)+\operatorname{dim} \xi\left(G_{4}\right)+\operatorname{dim} \xi\left(K_{m, n}\right)+\operatorname{dim} \xi\left(K_{n, p}\right)+ \\
& \operatorname{dim} \xi\left(K_{p, t}\right)+(m-1)+2(n-1)+2(p-1)+(t-1) \\
& =\left(q_{1}-m+1\right)+\left(q_{2}-n+1\right)+\left(q_{3}-p+1\right)+\left(q_{4}-t+1\right)+m n-(m+n)+1+n p-(n+p)+1+ \\
& p t-(p+t)+1+m-1+2 n-2+2 p-2+t-1 \\
& =\left(q_{1}+q_{2}+q_{3}+q_{4}+m n+n p+p t\right)-(m+n+p+t)+1 \\
& =\operatorname{dim} \xi\left(G_{1}+G_{2}+G_{3}+G_{4}\right) \text {. }
\end{aligned}
$$

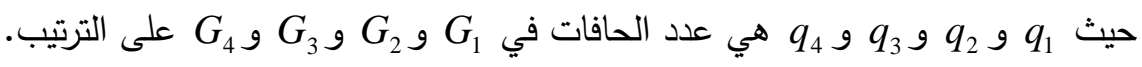

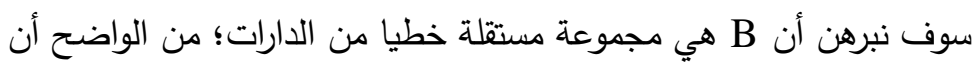
$B_{r}\left(G_{1}\right) \bigcup B_{r}\left(G_{2}\right) \bigcup B_{r}\left(G_{3}\right) \bigcup B_{r}\left(G_{4}\right) \bigcup B_{r}\left(K_{m, n}\right) \bigcup B_{r}\left(K_{n, p}\right) \bigcup B_{r}\left(K_{p, t}\right)$ مجموعة مستقلة خطيا وان كلاً من المجموعات

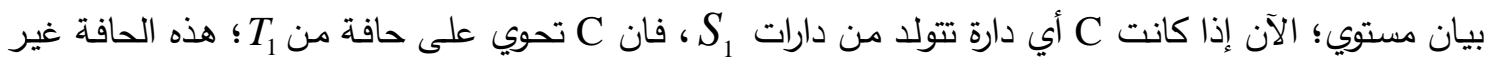

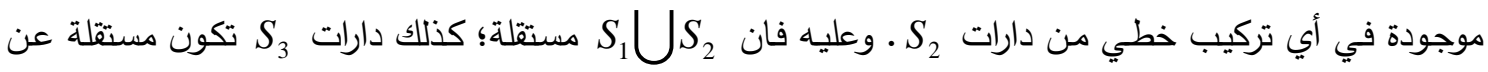
مات $S_{1} \bigcup S_{2}$ وعليـه فـان $S_{1} \bigcup S_{2}$ مان $S_{4} \bigcup S_{2} \bigcup S_{3}$ مسن $S=S_{1} \bigcup S_{2} \bigcup S_{3} \bigcup S_{4}$ $B^{\prime}=B_{r}\left(G_{1}\right) \bigcup B_{r}\left(G_{2}\right) \bigcup B_{r}\left(G_{3}\right) \bigcup B_{r}\left(G_{4}\right) \bigcup B_{r}\left(K_{m, n}\right) \bigcup B_{r}\left(K_{n, p}\right) \bigcup B_{r}\left(K_{p, t}\right)$

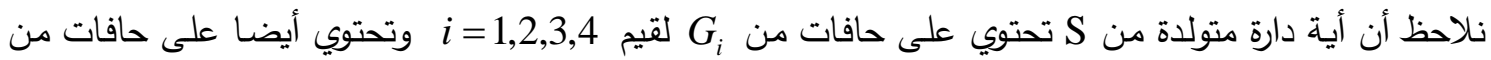
لقيم $K_{j, k}$ 

$$
\sum_{i=1}^{|S|} C_{i} \neq \sum_{j=1}^{\left|B^{\prime}\right|} b_{j} \quad, C_{i} \in S \quad \text { and } b_{j} \in B^{\prime}
$$

وبذلك تكون الآن نحسب الثية لكل حافة من حافات البيان؛ من (5)، الثثية لكل حافة من الحافات أدناه 


$$
\begin{array}{ll}
x_{i} y_{1}, x_{i} y_{n} & , i=2,3, \ldots, m-1, \\
x_{1} y_{j}, x_{m} y_{j} & , j=2,3, \ldots, n-1, \\
y_{1} z_{j}, y_{n} z_{j} & , j=2,3, \ldots, p-1, \\
y_{j} z_{1}, y_{j} z_{p} & , j=2,3, \ldots, n-1, \\
z_{1} u_{j}, z_{p} u_{j} & , j=2,3, \ldots, t-1, \\
z_{j} u_{1}, z_{j} u_{t} & , j=2,3, \ldots, p-1 .
\end{array}
$$

فـي القاعـدة 吕 $S_{1} \bigcup S_{2} \bigcup S_{3} \bigcup S_{4}$

$x_{1} y_{1}, x_{1} y_{n}, x_{m} y_{1}, x_{m} y_{n}, y_{1} z_{1}, y_{1} z_{p}, y_{n} z_{1}, y_{n} z_{p}, z_{1} u_{1}, z_{1} u_{t}, z_{p} u_{1}, z_{p} u_{t}$

هـي 1 فـي القاعـدة $)$ 列 $S_{1} \bigcup S_{2} \bigcup S_{3} \bigcup S_{4}$ $f_{B}(e) \leq 4, \forall e \in K_{m, n} \bigcup K_{n, p} \bigcup K_{p, t}$

إضافة لذلك من السهولة التحقق من أن $f_{B}(e) \leq \begin{cases}b\left(G_{1}\right)+1 & , \forall e \in G_{1}, \\ b\left(G_{2}\right)+2 & , \forall e \in G_{2}, \\ b\left(G_{3}\right)+2 & , \forall e \in G_{3}, \\ b\left(G_{4}\right)+1 & , \forall e \in G_{4} .\end{cases}$

max $\left\{4, b\left(G_{1}\right)+1, b\left(G_{2}\right)+2, b\left(G_{3}\right)+2, b\left(G_{4}\right)+1\right\}$ هي وبهذا يتم البرهان.

نتيجة (1):

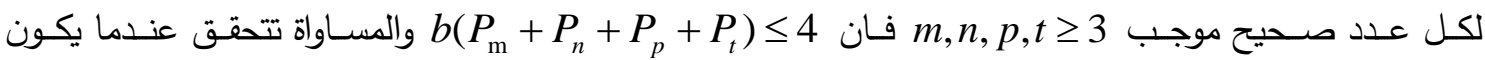
. $n, p \geq 6, m, t \geq 5$ البرهان :

من المبرهنة (1) (1) (1) (1)

$b\left(P_{\mathrm{m}}+P_{n}+P_{p}+P_{t}\right) \leq 4$

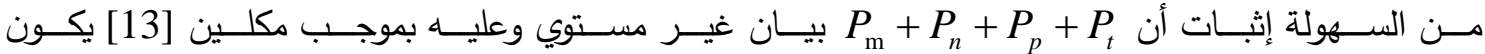
. $m, n, p, t \geq 3$ لقيم $b\left(P_{\mathrm{m}}+P_{n}+P_{p}+P_{t}\right) \geq 3$

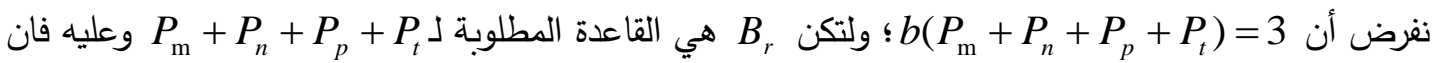
$\left|B_{r}\right|=\left|E\left(P_{\mathrm{m}}+P_{n}+P_{p}+P_{t}\right)\right|-\left|V\left(P_{\mathrm{m}}+P_{n}+P_{p}+P_{t}\right)\right|+1$ $\left|B_{r}\right|=m-1+n-1+p-1+t-1+m n+n p+p t-(m+n+p+t)+1$

$=m n+n p+p t-3$.

الآن عدد الدارات التي بطول 3 في القاعدة المطلوبة r هو $(m-1)+2(n-1)+2(p-1)+t-1=m+2 n+2 p+t-6$ 


$$
\sum_{i=1}^{\operatorname{dim} \xi(G)}\left|C_{i}\right| \leq b .|E(G)|,
$$

حيث | $\mid C_{i}$ يرمز إلى طول الدارة $4[(m n+n p+p t-3)-(m+2 n+2 p+t-6)]+3(m+2 n+2 p+t-6) \leq 3[(m-1)+$

$$
(n-1)+(p-1)+(t-1)+m n+n p+p t]
$$

$m n+n p+p t+6 \leq 4 m+5 n+5 p+4 t$

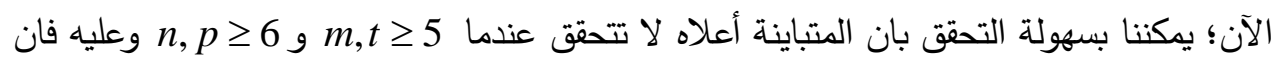
$b\left(P_{\mathrm{m}}+P_{n}+P_{p}+P_{t}\right) \geq 4 \quad \forall m, t \geq 5$ and $n, p \geq 6$

وبربط (6) مع (7) نحصل على المساواة.

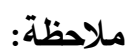

وبطريقة مشابهة لبرهان المبرهنة 1 نبرهن انه إذا كانت

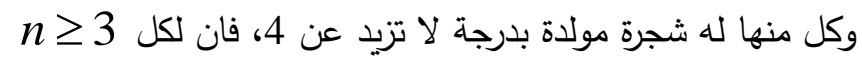
$b\left(G_{1}+G_{2}+\ldots+G_{n}\right) \leq \max \left\{4, \mathrm{~b}\left(\mathrm{G}_{1}\right)+1, \mathrm{~b}\left(\mathrm{G}_{\mathrm{n}}\right)+1, \mathrm{~b}\left(\mathrm{G}_{\mathrm{i}}\right)+2 \mid \mathrm{i}=2,3, \ldots, \mathrm{n}-1\right\}$. 


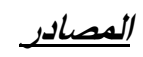

[1] Ali, A.A.; Marougi,G.T., (1993), The basis number of the lexicographic product of graphs, Ars combinatoria, Vol.36, pp.271-282.

[2] Ali, A.A.; Marougi,G.T., (1992), The basis number of the Cartesian product of some graphs, J. Indian Math. Soc., Vol. 58, No.2, pp. 123-134.

[3] Ali , A.A.,(1989), The basis number of the join of graphs ,Arab J. Maths., Vol. 10 No. $1 \& 2$, pp. 21-32.

[4] Alsardary, S.Y.; Ali, A.A., (2003), The basis number of some special non planar graphs, Czechoslovak Math. J., Vol. 53, No.2, pp. 225-240.

[5] Alzoubi, M.Y.; Jaradat, M.M., (2007), The basis number of the Cartesian product of a path with a circular ladder, a Möbius ladder and a net, Kyungpook Math. J., Vol. 47, No.2, pp. 165-174.

[6] Alzoubi, M.Y.; Jaradat, M.M., (2006), The basis number of the composition of theta graphs with some graph, Ars combinatoria, Vol.79, pp.107-114.

[7] Alzoubi, M.Y.; Jaradat, M.M., (2005), On the basis number of the composition of different ladders with some graphs, International Journal of Mathematics and Mathematical Sciences, Vol. 12, pp. 1861-1868.

[8] Banks, J.A.; Schmeichel, E.F., (1982), The basis number of the n-cube, J. combin. Theory, Ser. B, Vol. 33, No.2, pp. 95-100.

[9] Chartrand, G.; Lesniak, L., (1996), Graphs and Digraphs, 3d ed., Chapman \& Hall, CRC. Press.

[10] Harary, F., (1972), Graph Theory, $3^{\text {rd }}$ ed., Reading, Massachusetts, AddisonWesly.

[11] Jaradat, M.M.; Alzoubi, M.Y., (2005), An upper bound of the basis number of the lexicographic product of graphs, Australas J. comb., Vol. 32, pp. 305-312.

[12] Jaradat, M.M.; Alzoubi, M.Y.; Rawashdeh, E.A., (2004), The basis number of the lexicographic product of different ladders,SUT Journal of Mathematics, Vol. 40, No.2, pp. 91-101

[13] Maclane, S., (1937), A combinatorial condition for planar graphs, Fund. Math., Vol. 28, pp. 22-32.

[14] Marougi, G.T., (2009), On the basis number of semi-strong product of $K_{2}$ with some special graphs, Raf.J. of comp. \& Maths., Vol. 6, No.3, pp. 173-181.

[15] Marougi, G.T.,(2000), On the basis number of ternary join of graphs, Mu' tah Lil-Buhooth Wa Al-Dirasat Vol.15, No.1, pp.35-42.

[16] Schmeichel, E.F., (1981), The basis number of a graph, J. combin. Theory, Ser. B, Vol. 30, No.2, pp. 123-129. 